\title{
A Wearable Smart Device to Monitor Multiple Vital Parameters-VITAL ECG
}

\author{
Vincenzo Randazzo ${ }^{1, * \mathbb{D}}$, Jacopo Ferretti ${ }^{1,2}$ and Eros Pasero ${ }^{1, * \mathbb{D}}$ \\ 1 Politecnico di Torino, DET, 10129 Turin, Italy; jacopo.ferretti@unito.it \\ 2 Department of Surgical Sciences, Università degli Studi di Torino, 10126 Turin, Italy \\ * Correspondence: vincenzo.randazzo@polito.it (V.R.); eros.pasero@polito.it (E.P.)
}

Received: 23 January 2020; Accepted: 7 February 2020; Published: 9 February 2020

\begin{abstract}
Smart devices are more and more present in every aspect of everyday life. From smartphones, which are now like mini-computers, through systems for monitoring sleep or fatigue, to specific sensors for the recording of vital parameters. A particular class of the latter regards health monitoring. Indeed, through the use of such devices, several vital parameters can be acquired and automatically monitored, even remotely. This paper presents the second generation of VITAL-ECG, a smart device designed to monitor the most important vital parameters as a "one touch" device, anywhere, at low cost. It is a wearable device that coupled with a mobile app can track bio-parameters such as: electrocardiogram, $\mathrm{SpO}_{2}$, skin temperature, and physical activity of the patient. Even if it not yet a medical device, a comprehensive comparison with a golden standard electrocardiograph is presented to demonstrate the quality of the recorded signals and the validity of the proposed approach.
\end{abstract}

Keywords: mobile healthcare; telemedicine; ECG; EKG; electrocardiogram; pulse oximetry; wearable device; portable hospital; instrumentation amplifier; low-power sensors

\section{Introduction}

Pervasive dissemination of smart, low-cost, simple-to-use devices has deeply affected modern society. The continuously increasing availability of new sensors together with the development of new algorithms that analyze and learn from this data has led both scientists and companies to the creation of systems, which improve everyday life. Examples of such technologies are: smartphones, which are able to connect people all over the world and make them easily share contents, safety equipment for cars, and wearable devices for health monitoring.

A wide range of applications exploits smartphones and their set of embedded sensors (e.g., accelerometers or GNSS). Dominicis et al. [1] reported an example of an application of such paradigm. Smartphone-based systems are used for electrocardiogram (ECG) acquisition [2,3] and multichannel vital signal monitoring [4]. Crema et al. [5] present a solution that exploits smartphones to monitor driver vital parameters and measure his stress. In an alternative approach, wearable devices can be equipped with specific sensors [6,7]. For the same purpose, Deng et al. [8] propose a vision-based system.

Infrared cameras and motion sensor are used in Mandal et al. [9] to monitor sleep, detect sleep disorders and improve sleep quality. Alickovic and Subasi [10] applied support vector machines for automatic sleep stage classification. Axisa et al. [11] reported smart clothes for real time monitoring.

One of the most promising fields of application of such an approach is the telemedicine sector. The possibility of continuous patient monitoring while remotely performing a medical exam without the need of being inside of a hospital, opens tremendous scenarios for the future development of healthcare.

Today, there are no tools that allow physicians to remotely control patients' health or perform medical examinations. A representative example of this are the after-surgery hospital procedures. 
Indeed, even for basic surgeries, patients need to remain in hospital under medical observation to reduce the possibility of arising of any possible medical complication, which may lead to severe consequences $[12,13]$. At the current state of the art, it implies, even for small, easy surgeries, the need to remain in the hospital occupying a bed, which could, instead, being used for a subject with a more severe condition. While the person is in the hospital bed, several vital parameters are checked [14]. The main ones are:

- Heart activity

- Blood oxygen saturation

- Blood pressure

- Temperature

- Fatigue

- Perspiration

In addition, physicians may need to perform other more specific exams, e.g., blood tests or urinalysis, to gather a deeper insight about patient state of health $[15,16]$. Actually, in most cases, especially for day surgery, the exams listed above are enough to evaluate medical conditions and decide if a patient is likely to be discharged from hospitals without incurring any complication or if it is better to keep the subject under observation.

The current approach implies to have at the same time and in the same place:

- Multiple medical instrumentations, one for each parameter to be monitored (e.g., saturimeter, electrocardiograph, thermometer, etc.).

- Specialized personnel trained to execute properly the different exams and able to read its outcome (e.g., the ECG) correctly, that is, to make a valid diagnosis.

- A bed available for the patient.

Obviously, as per [17-20], also for basic surgery, the hospitalization cost per patient per day becomes quite high.

This work is an extension of our previous work [21] where the authors provided the initial experimental results of ECG WATCH, our first generation wireless device.

This paper presents the second generation of this device, see Figures 1 and 2, together with a complete comparison with a golden standard electrocardiograph.

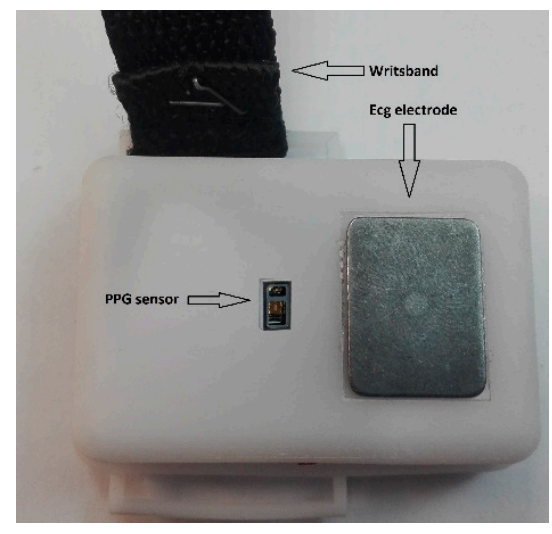

Figure 1. VITAL-ECG: top view. 


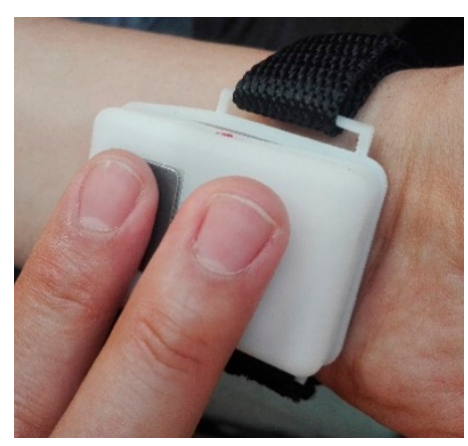

Figure 2. VITAL-ECG: acquisition in progress.

Section 2 presents the state of the art. Section 3 describes in detail the VITAL-ECG device. Section 4 shows the device testing. Finally, Section 5 yields the conclusions.

\section{State of the Art}

With respect to the state of the art, in order to be properly monitored, a patient should lie in a hospital bed while being connected to sophisticated machines [22], which are not portable or wearable, and whose outcome requires specialized personnel to be interpreted.

In healthcare facilities, the current medical standard requires ECG to be acquired using an electrocardiograph and ten wired electrodes, which record twelve leads at the same time. In the market are already present portable solutions that can record, simultaneously, a 12-lead ECG, filter it, and look for any alteration in the acquired signal [23-25]. They still use wired electrodes that should be applied by trained personnel. On the contrary, devices like [26] are wireless and do not need physicians to be used; unfortunately their acquisitions are of too low a quality to be used for diagnosis.

Pulse oximeters are either part of a multi-parameter station (heart rate, blood oxygen saturation, and temperature) or stand-alone finger devices (heart rate and $\mathrm{SpO}_{2}$ ). Finger devices can be either used in medical facilities or at patient home; can compute perfusion index and do not require wires; the more recent devices allow, also, to send data to mobile phone apps. Devices such as [27] are reliable enough to be used for diagnosis; however, due to the need of being worn on fingertips, they are not suited for continuous utilization and acquisition because it would be very uncomfortable for users.

Body temperature is acquired, at present, with digital readout thermometers or using bulb thermometers and manual readings. Then, temperature needs to be transcripted either on a paper or, more recently, digital, clinical folder, which prevents an automatic diagnosis.

Physical activity can be monitored with many non-medical devices, already available in the market, such as Fitbit [28]. Their outcome is, often, too approximated to estimate the amount of fatigue of a patient. They typically require system initialization and manual readings and transcription in the clinic folder. This approach is not, at all, automatic; then, the risk patients do not perform it as often as required is quite high. As a consequence, they cannot be classified as medical devices and recordings cannot be used for diagnosis.

VITAL-ECG presented in this paper was designed on a precise request of two Italian healthcare facilities. None of the mentioned approaches acquire all the vital parameters while, at the same time, being a wireless, wearable, friendly device for anytime, anywhere acquisition without the need of a physician or medical expertise.

\section{VITAL-ECG}

VITAL-ECG is a smart wristband developed by the Neuronica Lab of the Politecnico di Torino based on another device, previously designed in the same laboratory, called ECG-WATCH [29-31], which handles electrocardiograms.

VITAL-ECG is a device to acquire the main vital parameters such as heart rate and ECG, $\mathrm{SpO}_{2}$, skin temperature, and humidity. In addition, it also monitors the level of physical activity. It is low-cost 
and wearable. It uses a mobile app for a smartphone or tablet to store, visualize (see Figure 3), and analyze the acquired signals.

VITAL-ECG has been designed keeping particular attention to the ease of use. One of the main objectives, in fact, is to grant access to ECG measurement and to atrial fibrillation recognition without specialized expertise. Acquisitions are performed without the necessity of precise positioning or calibrations, while the only knowledge required is how to use an Android cellphone or tablet. When the device recognizes a hazardous situation, such as atrial fibrillation, a simple button on the app transmits patient location and data to a remote center, or to an assigned physician. In other words, VITAL-ECG is a tool for hospital to remotely monitor patients by means of real-time vital parameter acquisition and transmission, without the need of trained specialized personnel to be physically close to the subject. This telemedicine approach provides physicians a way to keep patient health under observation, remotely, without the need of physically occupying a bed in a hospital, and, only when needed, ask for their return to the medical facility for further exams.

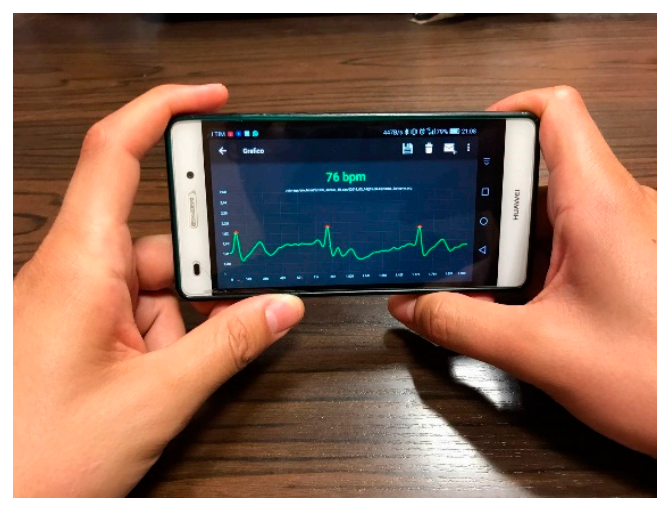

Figure 3. VITAL-ECG mobile app: ECG screen.

The Remote Assistance Center has a software complete with the required tools to assert the patient risk. Amongst the various functions, the tools allow to: view patient data, filter old records, compare acquisitions, analyze data, store notes, etc.

\subsection{System Design}

The device design specifications can be resumed in the following points:

- Two-electrode electrocardiograph. LEAD I, II, and III can be recorded one at a time.

- Atrial fibrillation monitor that once it detects an irregular rhythm automatically notifies the assigned physician.

- Temperature and relative humidity sensing.

- Gesture recognition sensor, used to count steps, and to wake up the device.

- Pulse oximetry, which can be used to improve heart-rate computation.

- Bluetooth 4.0+ to connect VITAL-ECG with the mobile app in low-energy.

- Very-low power consumption with a rechargeable lithium battery with standard USB Micro-B connector. A full charge should be sufficient for more than a week of normal use.

- Very simple use. Everything should work with the minimum effort possible.

- Comfortable design with contained dimension and weight.

- Biocompatibility: both the polycarbonate for the case and wristband, and the electrodes were chosen among those medical certified as biocompatible to prevent any harms to the user over long-term usage. 


\subsection{Circuit Design}

The overall VITAL-ECG hardware circuit is displayed in Figure 4 and its block diagram is shown in Figure 5. The only signal directly acquired by the microcontroller is the ECG, whilst the other sensor modules autonomously perform their acquisitions transmitting their data to the microcontroller only upon request. The microcontroller stores the received data and the acquired ECG in its internal FLASH memory until a Bluetooth device is ready to receive it. Once the data has been sent, the device automatically returns to a low-power state, in which it will remain until the next acquisition.

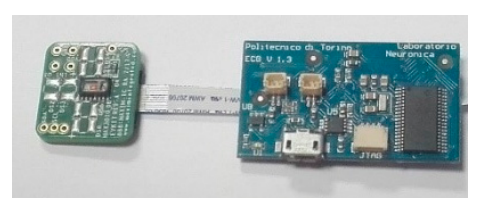

Figure 4. VITAL-ECG PCB.

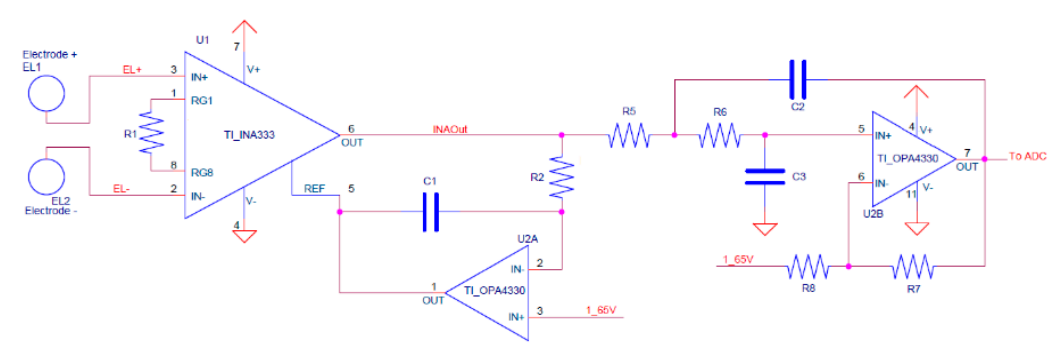

Figure 5. ECG analog front-end.

The system is intended to be as simple as possible in order to spare PCB space and power consumption. It also needs to be light and small enough to be placed into a wristwatch box. For this purpose, every component has been selected in its smallest and flattest package.

\subsection{ECG Front-End}

The ECG front-end schematic is shown in Figure 6. It consists of an instrumentation amplifier, which is directly connected to the electrodes, and two op-amps used as HP, and LP filters. The heart of the front-end is the instrumentation amplifier, implemented with the INA333 [32], from Texas Instruments, which has a very low power consumption of $150 \mu \mathrm{W}$, a CMRR higher than $100 \mathrm{~dB}$, and a built-in RFI filter. Furthermore, its relatively high resistance to ESD—4 kV HBM and $1 \mathrm{kV} \mathrm{CDM}$-allow to avoid the use of an ESD suppressor. An OPA4330 op-amp [33] has been chosen because of its low power consumption and high price-to-performance ratio.

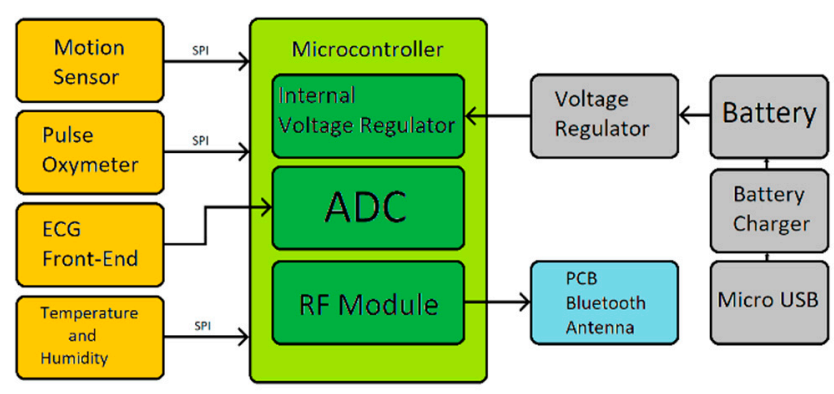

Figure 6. Block diagram representing the system architecture.

The HP filter is obtained with a single pole LP filter closed in loop to the INA333 reference. Since the circuit works with a monopolar power supply, a reference voltage of $1.65 \mathrm{~V}$ (half Vcc, obtained via decoupled voltage divider) had to be added as a reference. The second and final stage of the 
front-end is the LP filter, consisting of a Sallen-Key topology circuit designed using Butterworth polyonomy. The HP was set to $0.5 \mathrm{~Hz}$, while the LP was set to $40 \mathrm{~Hz}$. Further filtering of ECG signal is then performed with digital filters.

As stated in the last section, the circuit is not intended to be used during Bluetooth transmissions since it only transmits data after the end of acquisitions. However, having a $8 \mathrm{MHz}$ corner frequency, the INA333's built-in RFI filters are enough to highly reduce any possible interference with the Bluetooth RF front-end, or any similar frequency signal (e.g., Wi-Fi). Any remaining interference is then filtered by the LP filter with over $-200 \mathrm{~dB}$ attenuation for frequencies over $4 \mathrm{MHz}$. As a matter of fact, all the acquisitions ever made with VITAL-ECG are performed in presence of multiple mobile phones-and any sort of connected devices nearby — without showing any sign of signal degradation.

\subsection{Electrodes}

Since one of the main goals of VITAL-ECG is the ease of use, dry electrodes were the only logical choice. Wet electrodes require gels, or disposable elements, producing garbage, and making the ECG acquisition considerably more uncomfortable. On the other hand, dry electrodes have poor performance when compared to wet ones. Furthermore, they are usually made of expensive metals, like silver, that also tend to oxidize easily. Albeit it is unquestionable that wet electrodes offer better performance over dry ones, their discomfort in this kind of device does not justify the improvement of the signal, which is already acceptable — for the purpose of the VITAL-ECG—with rigid metal electrodes. In order to select the best electrode material, in terms of cost and performance, several experiments were carried out on different materials, such as silver, copper, heavily oxidized silver, heavily oxidized copper, gold, and steel. Although in the end stainless steel was chosen as the best compromise, further tests are required in order to find a better solution. Finally, stainless steel provides biocompatibility over long-term usage on the wrist; indeed, it is one of the standard materials for building watches.

\subsection{Pulse Oximeter}

Pulse oximetry is a non-invasive measurement of a person's peripheral oxygen saturation $\left(\mathrm{SpO}_{2}\right)$ [34]. The typical configuration of a pulse oximeter requires two LEDs, one emitting red light, and the other one infrared light; and a photodiode, which measures the reflected light from the patient.

In the VITAL-ECG, pulse oximetry is implemented with a MAX30102 [35]. The main advantage of using an integrated circuit is that the LEDs and photodiode are embedded in the same die, as well as the optics required to work. This allows a drastic reduction in PCB space and, most importantly, the manufacturing complexity of the final board. Another advantage of the integrated solution is that acquisition, post-processing, and $\mathrm{SpO}_{2}$ calculation are all performed before being sent to the microcontroller, which then will require no further operation on the data.

\subsection{Temperature and Humidity}

Temperature and humidity are both acquired by an HTS221 [36]. This IC is a factory calibrated, low power, ultra-compact sensor that contains a 16-bit ADC, and communicate with the microcontroller via SPI.

\subsection{Motion Sensor}

The motion sensing in VITAL-ECG is obtained with an MPU-9250 [37]. This is a nine-axis motion tracking device that combines a three-axis accelerometer, three-axis magnetometer, three-axis gyroscope and a digital motion processor (DMP) all in a small QFN package. It contains nine 16-bit ADCs (one for each axis), and programmable digital filters. The DMP allows to perform basic gesture recognition, such as tilts, and is capable of triggering interrupts with a dedicated pin. 
The main use of the motion sensor is step counting, and to wake up the device. However, in the future it could be possible to implement advanced gesture recognition to assert hazardous events, such as falling.

\subsection{Power}

The device is powered by a single $3.7 \mathrm{~V}$ LiPo battery with $190 \mathrm{mAh}$ capacity. The battery voltage is then regulated at $3.3 \mathrm{~V}$ with the MAX1759 [38], which is a low noise buck-boost voltage converter that does not require an external inductor, thus minimizing PCB space.

The power circuitry section also includes the MAX1555 [39], which is an integrated circuit that allows to safely charge the battery taking care of the required protection, such as overcharging, short-circuit, overheat, polarity exchange, etc. The battery is charged through a standard USB type micro-B connector, which requires the same cable used to charge modern cellphones ensuring maximum versatility.

The total power consumption of the device depends on its use, which may vary from an average value of around $160 \mu \mathrm{W}$ (measured value), when in standby, to an average value of $30 \mathrm{~mW}$ (measured value) when transmitting. In a normal use scenario (no more than three acquisition per day), this would optimistically allow recharging the battery around once every two months.

\subsection{Microcontroller}

The microcontroller chosen for VITAL-ECG is the CC2640R2F, from Texas Instruments [40]. This microcontroller is specifically designed for low power wireless sensing applications. In fact, its very low power consumption ranges from $9 \mathrm{~mW}$ in full speed operation, to $2 \mu \mathrm{W}$ in standby. To further improve its power consumption, this microcontroller contains an ultra-low power sensor controller that allows the integrated 12 bit ADC-amongst other peripherals-to work autonomously from the $\mathrm{CPU}$, which can be selectively disabled to save energy during acquisitions. Finally, the main reason why it was chosen is the integrated Bluetooth transceiver, which only requires a small patch antenna in order to work, hence a very small PCB area and implies also an easier implementation.

In order to improve battery performances, the CC2640R2F is put in shut-down mode whenever possible. For this purpose, the MPU-9250 is exploited to awaken the device when the user taps three times on the top electrode.

\subsection{Bluetooth}

The integrated RF transceiver in CC2640R2F is compatible with Bluetooth low energy (BLE) 4.2 and 5.0 specifications [41].

It is extremely unlikely that VITAL-ECG will require long range Bluetooth transmissions since the form factor is a watch. For this reason, it was possible to design the antenna as a low gain patch antenna, hence obtained with a PCB trace. This not only ensures the minimum space occupation on the board, but it also limits the total power consumption of the device to less than $13 \mathrm{~mW}$ during transmission. In order to further reduce power consumptions, Bluetooth communication is limited to a single transmission burst at the end of the acquisition, besides the unavoidable start signal.

\section{Experiments}

To assess the quality of the VITAL-ECG, its ECG acquisitions have been compared with those of a professional electrocardiograph on 36 healthy subjects. The system chosen as reference is the General Electric MAC2000 (Boston, USA) [42]: an electrocardiograph widely used in hospitals and known for its reliability. The experiments consisted in five contemporary acquisitions of VITAL-ECG and MAC2000 for each subject. All data obtained was then analyzed to assess potential differences in the acquisitions.

The MAC2000 was equipped with four stainless steel electrodes-the same used in the VITAL-ECG - and they were placed on the patient according to Einthoven's triangle: right hand, left hand, and left leg [43], while the fourth is placed on the right leg to reduce interferences. This configuration 
allows to obtain three channel acquisitions: LEAD I, LEAD II, and LEAD III. However, since most of the acquisitions using VITAL-ECG are taken from the left hand to the right hand, only LEAD I is taken in consideration for the results analysis. In fact, for the experiments here proposed the acquisitions of VITAL-ECG were performed between the right wrist and the left thumb, thus obtaining a LEAD I signal.

Since the purpose of this analysis is to assert the quality of the proposed device, all the results shown in this paper are obtained from healthy resting subjects, thus avoiding all kind of movements that would introduce unnecessary motion artifacts. The experiments start with the patient seated and in a relaxed condition. Then five series of ten second acquisitions are performed interspersed with one minute of rest, during which data is saved and the following acquisition is set up.

Through the experiments, the device has also been stressed to test its resilience to alterations both in the working environment, e.g., electromagnetic fields, and the patient, e.g., after some sports. In both cases, the system has not shown any significant modification in the acquired signal, which was not automatically removed during the app filtering.

Finally, in one-third of the experiments, the volunteers have been asked to wear the device an whole day and to perform as much acquisitions as possible to check if the acquired signal were consistent over time or if, on the contrary, they were affected by some noise. The resulting VITAL-ECG recordings did not show any alteration except in case of battery discharge; at this purpose, a firmware routine has been added to check battery voltage, to block acquisitions when this kind of situation occurs and to signal it to the app and, consequently, to the user.

\subsection{Bland-Altman Plot}

The first parameter that was taken into account in order to compare VITAL-ECG and MAC2000 ECG's quality is the heart-rate estimation through a Bland-Altman plot. Bland-Altman plots are normally used to compare two measurement systems by plotting differences between the same measurement in the ordinate, and its average on the abscissa.

Figure 7 presents the Bland-Altman plot for the VITAL-ECG and MAC2000, where each blue point corresponds to a pair of heart-rate measurements, represented by their difference and mean. The plot exhibits a zero-mean value, with less than $5 \%$ of data variation around the maximum, which means that on average there is no difference between the heart-rate measured by the two systems. Furthermore, cross-correlation (XC) between the two heart-rate detections has been evaluated. The results confirm measurement's consistency with XC close to $90.5 \%$. As a consequence, VITAL-ECG heartbeat measurements are consistent and it can be stated the device correctly estimates the heartbeat.

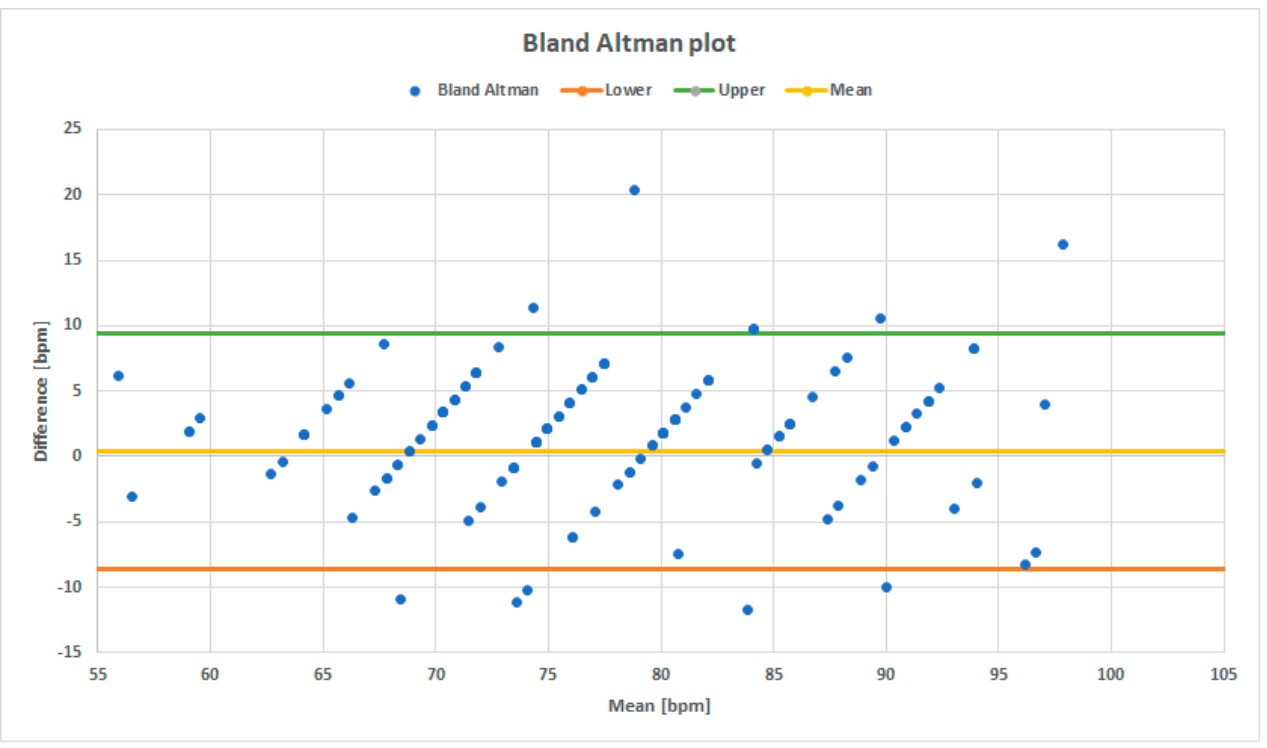

Figure 7. Bland-Altman plot for the VITAL-ECG and MAC2000. 


\subsection{Power Spectral Density}

The second parameter investigated to assert VITAL-ECG's quality is the ECG signal itself.

Albeit electrocardiogram is a quite old and very-well known topic in literature, asserting its quality is not a trivial task, especially when an analytical standpoint is required. A study about the ECG signal manifold and intrinsic dimensionality is presented in [44]. In the following, both frequency and time domain analyses have been used to evaluate ECG signals.

Power spectral density (PSD) represents how the signal's energy is distributed among the frequency spectrum, which is also correlated to the information content of the signal among the spectrum. Although there are many different methods better suited to estimate PSD, for simplicity's sake in this study the squared discrete fast Fourier transform (FFT) module has been chosen.

$$
\operatorname{PSD}(f)=\frac{(\Delta t)^{2}}{T}\left|\sum_{n=1}^{N} x_{n} e^{-i \omega n \Delta t}\right|^{2}
$$

Results of the PSD are shown in Figure 8, where the red line represents the VITAL-ECG and the green line represents the MAC2000.

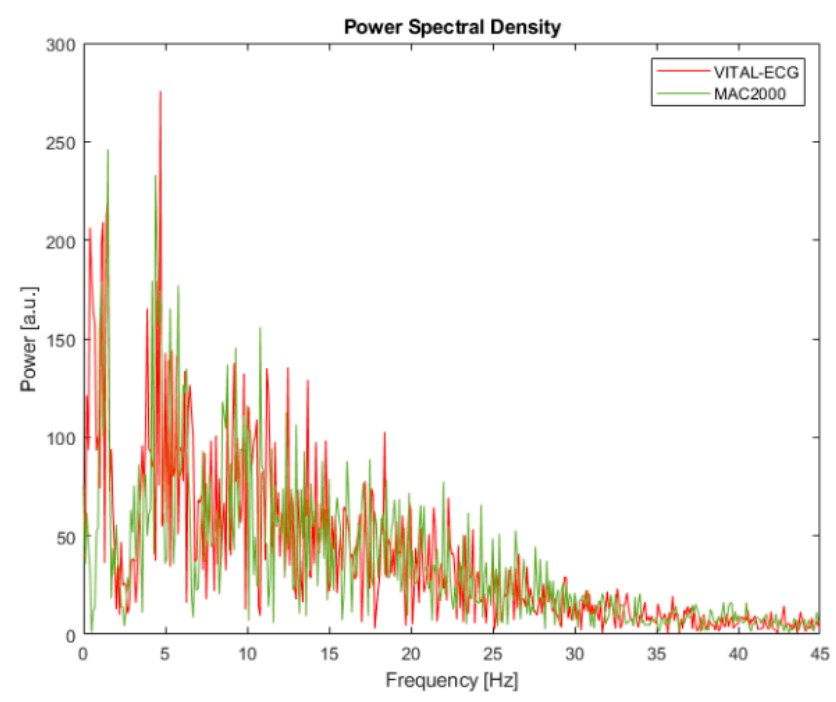

Figure 8. Power spectral density (PSD) between 0 and $45 \mathrm{~Hz}$ for VITAL-ECG (red) and MAC2000 (green).

Although by visual inspection there is no significant difference between the two PSDs, a further analytical analysis was held through cumulative spectral power.

Cumulative spectral power (CSP) is a parameter calculated from PSD with a cumulative sum normalized with the total power. The curve obtained in this way, CSP(f), is a monotonously raising function representing the percentage of energy contained by the frequencies below a certain frequency of interest $\mathrm{f}$.

$$
\operatorname{CSP}(f)=\sum_{n=1}^{f} \operatorname{PSD}(n)
$$

According to the argument of this function, it is possible to assess at which frequency the signals arrive to a certain fraction of total power, and thus of the information content. Therefore, it is possible to define the median of the PSD, namely the frequency that divides the power in half, and a certain bandwidth around the median, which has been chosen to $60 \%$ for this study. In Table 1 there are reported the frequencies at which $20 \%, 50 \%$, and $80 \%$ of the total power is distributed, according to CSP. 
Table 1. Cumulative spectrum power frequencies.

\begin{tabular}{cccc}
\hline System & $\mathbf{f} \mathbf{2 0} \mathbf{\%} \mathbf{( H z )}$ & $\mathbf{f ~} \mathbf{5 0} \mathbf{~ ( H z )}$ & $\mathbf{f} \mathbf{8 0} \mathbf{~ ( H z )}$ \\
\hline MAC2000 & 5.5 & 13.6 & 31.9 \\
Vital-ECG & 4.3 & 11.4 & 25.6 \\
\hline
\end{tabular}

The values in Table 1 confirm that the information of the VITAL-ECG and MAC2000 is distributed in a very similar matter, in accordance with Figure 8. In particular, half of the total information content of VITAL-ECG acquisitions is concentrated in the bandwidth 0-11.4 Hz, which means that its spectrum is concentrated on slightly lower frequencies than MAC2000 (0-13.6 Hz). Since most of ECG information is located on low frequencies [45], Table 1 shows that the VITAL-ECG has better performance in that bandwidth.

\subsection{Time Domain Differences}

The last comparison between the VITAL-ECG and MAC2000 is in the time domain. For this analysis, a set of various single heartbeats has been built from random subjects in order to evaluate point-to-point differences between the measurement systems. For this purpose, the contemporary acquired signals from the MAC2000 and VITAL-ECG were first normalized, then matching heartbeats were isolated and compared in couples. Figure 9 shows an example of the heartbeat acquired with both the VITAL-ECG (in blue) and MAC2000 (in red).

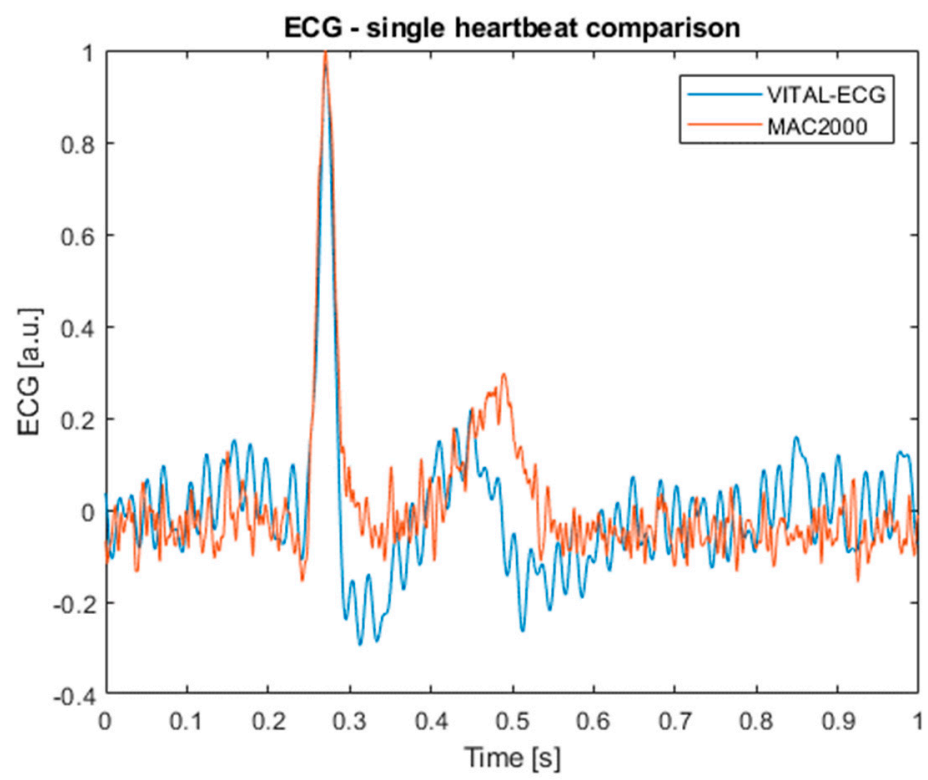

Figure 9. A single heartbeat isolated from VITAL-ECG and MAC2000 contemporary acquisition.

Table 2 shows the mean difference and the standard deviation between each point of the signal. Even from this table it is possible to observe that there are not important disagreements, with a mean difference around 1\%, and a standard deviation slightly above $12 \%$. Table 2 further confirms the previous analyses, showing no significant differences between the acquisitions of the two devices.

Table 2. Mean and standard deviation of time domain differences.

\begin{tabular}{ccc}
\hline & Mean (a.u.) & Standard Deviation (a.u.) \\
\hline Difference & -0.011 & 0.1213 \\
\hline
\end{tabular}

As a final note, it is important to remark that although is not very evident from the final ECG signals, the MAC2000 caused a significant $40 \mathrm{~Hz}$ interference on the VITAL-ECG acquisitions, probably 
due to the right leg driver. For this reason, the spectral analysis had to be done on signals acquired without its presence. All other measurements were held using contemporary acquisitions.

\section{Conclusions}

To ensure patients are healthy, several vital parameters need to be kept under physician control. These operations imply the use of various medical instrumentations and need a free bed in the hospital. Furthermore, results need to be interpreted by specialized personnel, which need to be physically near the instrumentation at hand. Today, no such portable or wearable solution exists. The second generation of the VITAL-ECG has been presented. After a detailed and rigorous description of the circuit, a comparison with the MAC2000 electrocardiograph has demonstrated the correctness of the adopted approach and its physical implementation. In this sense, the VITAL-ECG is able to perform different medical exams, which need, in general, different instrumentations, within a single device. As a matter of fact, the analyses presented in the paper showed no significant difference in terms of the measured heart-rate, spectral content of information, and time domain differences.

Future works will deal with increasing the number of sensors to make the device able to record also perspiration, blood pressure, and patient stress $/ \mathrm{mood}$. To memorize data directly in the device, an external peripheral, such as an SD card, will be mounted; as a consequence, the VITAL-ECG would also be used to carry out continuous monitoring of subjects at risk. To improve the user experience, the device will be equipped with a display. Finally, the heartbeat algorithm will be improved to further reduce its uncertainty.

\section{Patents}

The device presented here is based on patent WO2018073847A1: Wearable device for acquiring electrocardiographic signals (ECG) signals.

Author Contributions: According to the journal proposed scheme (https://img.mdpi.org/data/contributor-roleinstruction.pdf), the authors have contributed in the following way: Conceptualization: E.P. and V.R.; Methodology: E.P. and J.F.; Software: V.R.; Validation: V.R., J.F. and E.P.; Formal analysis: J.F.; Investigation: J.F.and V.R.; Resources: E.P. and V.R.; Data curation: J.F.and V.R.; Writing—original draft preparation: V.R. and J.F.; Writing一review and editing: E.P., J.F. and V.R.; Visualization: E.P.; Supervision: E.P.; Project administration: E.P. and V.R.; Funding acquisition: E.P. and V.R. All authors have read and agreed to the published version of the manuscript.

Funding: This work has been partly supported by the "Proof of Concept" project of the Politecnico di Torino and the PoliToBIOMed Lab - Biomedical Engineering Lab of the Politecnico di Torino.

Acknowledgments: A special thanks to Federico Caffarelli and Giacomo Zanichelli.

Conflicts of Interest: The authors declare no conflict of interest. The funders had no role in the design of the study; in the collection, analyses, or interpretation of data; in the writing of the manuscript; or in the decision to publish the results.

\section{References}

1. De Dominicis, C.M.; Mazzotti, D.; Piccinelli, M.; Rinaldi, S.; Vezzoli, A.; Depari, A. Evaluation of Bluetooth Hands-Free profile for sensors applications in smartphone platforms. In Proceedings of the 2012 IEEE Sensors Applications Symposium Proceedings; Institute of Electrical and Electronics Engineers (IEEE), Brescia, Italy, 7-9 February 2012; pp. 1-6.

2. Depari, A.; Flammini, A.; Sisinni, E.; Vezzoli, A. A wearable smartphone-based system for electrocardiogram acquisition. In Proceedings of the 2014 IEEE International Symposium on Medical Measurements and Applications (MeMeA), Lisboa, Portugal, 11-12 June 2014; pp. 1-6.

3. De Dominicis, C.; Depari, A.; Flammini, A.; Rinaldi, S.; Sisinni, E.; Vezzoli, A. Acquisition and elaboration of cardiac signal in android Smartphone devices. In Proceedings of the 2014 IEEE Sensors Applications Symposium (SAS), Queenstown, New Zealand, 18-20 February 2014; pp. 83-88.

4. Crema, C.; Depari, A.; Flammini, A.; Sisinni, E.; Vezzoli, A. The WearPhone: Changing smartphones into multichannel vital signs monitors. In Proceedings of the 2016 IEEE International Symposium on Medical Measurements and Applications (MeMeA), Benevento, Italy, 15-18 May 2016; pp. 1-6. 
5. Crema, C.; Depari, A.; Flammini, A.; Vezzoli, A.; Benini, C.; Chindamo, D.; Gadola, M.; Romano, M. Smartphone-based system for the monitoring of vital parameters and stress conditions of amatorial racecar drivers. In Proceedings of the 2015 IEEE SENSORS, Busan, South Korea, 1-4 November 2015; pp. 1-4.

6. Muhlbacher-Karrer, S.; Mosa, A.H.; Faller, L.-M.; Ali, M.; Hamid, R.; Zangl, H.; Kyamakya, K. A Driver State Detection System-Combining a Capacitive Hand Detection Sensor with Physiological Sensors. IEEE Trans. Instrum. Meas. 2017, 66, 1-13. [CrossRef]

7. Choi, M.; Koo, G.; Seo, M.; Kim, S.W. Wearable Device-Based System to Monitor a Driver's Stress, Fatigue, and Drowsiness. IEEE Trans. Instrum. Meas. 2018, 67, 634-645. [CrossRef]

8. Deng, F.; Dong, J.; Wang, X.; Fang, Y.; Liu, Y.; Yu, Z.; Liu, J.; Chen, F. Design and Implementation of a Noncontact Sleep Monitoring System Using Infrared Cameras and Motion Sensor. IEEE Trans. Instrum. Meas. 2018, 67, 1555-1563. [CrossRef]

9. Mandal, B.; Li, L.; Wang, G.S.; Lin, J. Towards Detection of Bus Driver Fatigue Based on Robust Visual Analysis of Eye State. IEEE Trans. Intell. Transp. Syst. 2017, 18, 545-557. [CrossRef]

10. Aličković, E.; Subasi, A. Ensemble SVM Method for Automatic Sleep Stage Classification. IEEE Trans. Instrum. Meas. 2018, 67, 1258-1265. [CrossRef]

11. Axisa, F.; Dittmar, A.; Delhomme, G. Smart clothes for the monitoring in real time and conditions of physiological, emotional and sensorial reactions of human. In Proceedings of the 25th Annual International Conference of the IEEE Engineering in Medicine and Biology Society (IEEE Cat. No.03CH37439), Cancun, Mexico, 17-21 September 2003; Volume 4, pp. 3744-3747.

12. Verma, R.; Alladi, R.; Jackson, I.; Johnston, I.; Kumar, C.; Page, R.; Smith, I.; Stocker, M.; Tickner, C.; Williams, S.; et al. Day case and short stay surgery: 2. Anaesthesia 2011, 66, 417-434.

13. WHO. Guidelines for Safe Surgery: Safe Surgery Saves Lives; World Health Organization: Geneva, Switzerland, 2009.

14. Quemby, D.J.; Stocker, M.E. Day surgery development and practice: Key factors for a successful pathway. Contin. Educ. Anaesth. Crit. Care Pain 2014, 14, 256-261. [CrossRef]

15. Hamer, C.; Holmes, K.; Stocker, M. A generic process for transferring procedures to the day case setting: The Torbay hospital proposal. J. One-Day Surg. 2008, 18, 9-12.

16. Wilmore, D.W.; Kehlet, H. Recent advances: Management of patients in fast track surgery. BMJ 2001, 322, 473-476. [CrossRef] [PubMed]

17. Hospital Adjusted Expenses per Inpatient Day by Ownership. In 1999-2015 AHA Annual Survey; AHA: Dallas, TX, USA, 2016.

18. Epelde, F.; Iglesias-Lepine, M.; Anarte, L. Economic crisis: Cost and effectiveness of short stay hospital units. An. Sist. Sanit. de Navar. 2012, 35, 469-475. [CrossRef]

19. Tolou-Ghamari, Z.; Shaygannejad, V.; Khorvash, F. Preliminary Investigation of Economics Issues in Hospitalized Patients with Stroke. Int. J. Prev. Med. 2013, 4, S338-S342. [PubMed]

20. Latta, V.B.; Keene, R.E. Use and cost of short-stay hospital inpatient services under Medicare, 1988. Healthc. Financ. Rev. 1990, 12, 91-99.

21. Randazzo, V.; Pasero, E.; Navaretti, S. VITAL-ECG: A portable wearable hospital. In Proceedings of the 2018 IEEE SENSORS, Seoul, South Korea, 12-14 March 2018; pp. 1-6.

22. CREATIVE MEDICAL Monitor Multiparametrico UP7000. Available online: https://www.doctorshop.it/ Prodotti/apparecchiature-per-lemergenza-PS-1/monitor-multiparametrici-e-accessori-PS-1-03/monitormultiparametrici-PS-1-03-01/monitor-multiparametrico-up7000-spo2-nibptemprespecg-7-derivazioni106496 (accessed on 7 February 2020).

23. ECG CONTEC 1200G-12 Canali con Display. Available online: http://www.gimaitaly.com/prodotti.asp?sku= 33223\&dept_selected=5802\&dept_id=5802 (accessed on 7 February 2020).

24. ECG CARDIO 7 (ECG 12 CANALI CON TOUCH SCREEN). Available online: http://www.gimaitaly.com/ prodotti.asp?sku=33357\&dept_selected=5802\&dept_id=5802 (accessed on 7 February 2020).

25. ECG CARDIOGIMA 12-3/6/12 Canali. Available online: http://www.gimaitaly.com/prodotti.asp?sku=33354\& dept_selected=5802\&dept_id=5802 (accessed on 7 February 2020).

26. ECG PALMARE PM10. Available online: http://www.gimaitaly.com/prodotti.asp?sku=33246\&dept selected=5801\&dept_id=5801 (accessed on 7 February 2020).

27. iHEALTH AIR-PULSOXIMETRO WIRELESS. Available online: http://www.gimaitaly.com/prodotti.asp? sku=23525\&dept_selected=82\&dept_id=82 (accessed on 7 February 2020).

28. FitBit. Available online: https://www.fitbit.com/it/home (accessed on 7 February 2020). 
29. Lacirignola, F.; Pasero, E. Hardware design of a wearable ECG-sensor: Strategies implementation for improving CMRR and reducing noise. In Proceedings of the 2017 European Conference on Circuit Theory and Design (ECCTD); Institute of Electrical and Electronics Engineers (IEEE), Catania, Italy, 4-6 September 2017; pp. 1-4.

30. Pasero, E.; Balzanelli, E.; Caffarelli, F. Intruder recognition using ECG signal. In Proceedings of the 2015 International Joint Conference on Neural Networks (IJCNN); Institute of Electrical and Electronics Engineers (IEEE), Killarney, Ireland, 12-17 July 2015; pp. 1-8.

31. Randazzo, V.; Ferretti, J.; Pasero, E. ECG WATCH: A real time wireless wearable ECG. In Proceedings of the 2019 IEEE International Symposium on Medical Measurements and Applications (MeMeA), Istanbul, Turkey, 26-28 June 2019; pp. 1-6.

32. INA333: Low-Power, Zero-Drift, Precision Instrumentation Amplifier. Available online: http://www.ti.com/ product/INA333 (accessed on 7 February 2020).

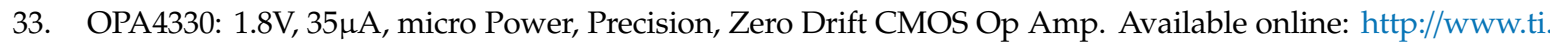
com/product/OPA4330 (accessed on 7 February 2020).

34. Nakajima, S. Clinical application of a new (fingertip type) pulse wave oximeter. (Translated from the Japanese). Jpn. J. Surg. 1979, 41, 57-61.

35. MAX30102: High-Sensitivity Pulse Oximeter and Heart-Rate Sensor for Wearable Health, Maximintegrated. Available online: https://www.maximintegrated.com/en/products/interface/sensor-interface/MAX30102.html (accessed on 7 February 2020).

36. HTS221: Capacitive Digital Sensor for Relative Humidity and Temperature, St. Available online: http: //www.st.com/en/mems-and-sensors/hts221.html (accessed on 7 February 2020).

37. MPU-9250 Nine-Axis (Gyro + Accelerometer + Compass) MEMS MotionTracking ${ }^{\mathrm{TM}}$ Device, Invesens. Available online: https://www.invensense.com/products/motion-tracking/9-axis/mpu-9250/ (accessed on 7 February 2020).

38. MAX1759: Buck/Boost Regulating Charge Pump in $\mu \mathrm{MAX}$, Maximintegrated. Available online: https: //www.maximintegrated.com/en/products/MAX1759 (accessed on 7 February 2020).

39. MAX1555: SOT23, Dual-Input, USB/AC Adapter, 1-Cell Li+ Battery Chargers, Maximintegrated. Available online: https://www.maximintegrated.com/en/products/power/battery-management/MAX1555. html (accessed on 7 February 2020).

40. CC2640R2F: SimpleLink Bluetooth ${ }^{\circledR}$ Low Energy Wireless MCU, Texas Instruments. Available online: http://www.ti.com/product/CC2640R2F (accessed on 7 February 2020).

41. Bluetooth Specifications, Bluetooth Market Update. Available online: https://www.bluetooth.com/ specifications (accessed on 7 February 2020).

42. MAC2000: Resting ECG System Overview, General Electric. Available online: http://www3.gehealthcare.it/ it-it/prodotti/categorie/diagnostic_cardiology/resting_ecgs/mac_2000 (accessed on 7 February 2020).

43. Butterworth, J.S.; Thorpe, J.J. On Evaluating the Einthoven Triangle Theory. Circulation 1951, 3, $923-925$. [CrossRef] [PubMed]

44. Bland, D.G.A.M. Measurement in Medicine: The Analysis of Method Comparison Studies. J. R. Stat. Soc. Ser. D 1983, 32, 307.

45. Scher, A.M.; Young, A.C. Frequency Analysis of the Electrocardiogram. Circ. Res. 1960, 8, 344-346. [CrossRef] [PubMed]

(C) 2020 by the authors. Licensee MDPI, Basel, Switzerland. This article is an open access article distributed under the terms and conditions of the Creative Commons Attribution (CC BY) license (http://creativecommons.org/licenses/by/4.0/). 